\title{
A Note on Jordan Triple Higher $*$-Derivations on Semiprime Rings
}

\author{
O. H. Ezzat \\ Mathematics Department, Al-Azhar University, Nasr City, Cairo 11884, Egypt \\ Correspondence should be addressed to O. H. Ezzat; ohezzat@yahoo.com
}

Received 9 February 2014; Accepted 26 March 2014; Published 9 April 2014

Academic Editors: E. Aljadeff, A. Jaballah, A. Kılıçman, F. Kittaneh, and H. You

Copyright (C) 2014 O. H. Ezzat. This is an open access article distributed under the Creative Commons Attribution License, which permits unrestricted use, distribution, and reproduction in any medium, provided the original work is properly cited.

We introduce the following notion. Let $\mathbb{N}_{0}$ be the set of all nonnegative integers and let $D=\left(d_{i}\right)_{i \in \mathbb{N}_{0}}$ be a family of additive mappings of a $*$-ring $R$ such that $d_{0}=i d_{R} ; D$ is called a Jordan higher $*$-derivation (resp., a Jordan higher $*$-derivation) of $R$ if $d_{n}\left(x^{2}\right)=$ $\sum_{i+j=n} d_{i}(x) d_{j}\left(x^{*^{i}}\right)$ (resp., $\left.d_{n}(x y x)=\sum_{i+j+k=n} d_{i}(x) d_{j}\left(y^{*^{i^{i}}}\right) d_{k}\left(x^{*^{i+j}}\right)\right)$ for all $x, y \in R$ and each $n \in \mathbb{N}_{0}$. It is shown that the notions of Jordan higher $*$-derivations and Jordan triple higher $*$-derivations on a 6 -torsion free semiprime $*$-ring are coincident.

\section{Introduction}

Let $R$ be an associative ring, for any $x, y \in R$. Recall that $R$ is prime if $x R y=0$ implies $x=0$ or $y=0$ and is semiprime if $x R x=0$ implies $x=0$. Given an integer $n \geq 2, R$ is said to be $n$-torsion free if, for $x \in R, n x=0$ implies $x=0$. An additive mapping $x \rightarrow x^{*}$ satisfying $(x y)^{*}=y^{*} x^{*}$ and $\left(x^{*}\right)^{*}=x$ for all $x, y \in R$ is called an involution and $R$ is called a $*$-ring.

An additive mapping $d: R \rightarrow R$ is called a derivation if $d(x y)=d(x) y+y d(x)$ holds for all $x, y \in R$, and it is called a Jordan derivation if $d\left(x^{2}\right)=d(x) x+x d(x)$ for all $x \in R$. Every derivation is obviously a Jordan derivation and the converse is in general not true [1, Example 3.2.1]. An influential Herstein theorem [2] shows that any Jordan derivation on a 2-torsion free prime ring is a derivation. Later on, Brešar [3] has extended Herstein's theorem to 2torsion free semiprime rings. A Jordan triple derivation is an additive mapping $d: R \rightarrow R$ satisfying $d(x y x)=d(x) y x+$ $x d(y) x+x y d(x)$ for all $x, y \in R$. Any derivation is obviously a Jordan triple derivation. It is also easy to see that every Jordan derivation of a 2-torsion free ring is a Jordan triple derivation [4, Lemma 3.5]. Brešar [5] has proved that any Jordan triple derivation of a 2-torsion free semiprime ring is a derivation.

Let $R$ be a $*$-ring. An additive mapping $d: R \rightarrow R$ is called a $*$-derivation if $d(x y)=d(x) y^{*}+x d(y)$ holds for all $x, y \in R$, and it is called a Jordan $*$-derivation if $d\left(x^{2}\right)=d(x) x^{*}+x d(x)$ holds for all $x \in R$. We might guess that any Jordan $*$-derivation of a 2 -torsion free prime $*$-ring is a $*$-derivation, but this is not the case. It has been proved in [6] that noncommutative prime $*$-rings do not admit nontrivial $*$-derivations. A Jordan triple $*$-derivation is an additive mapping $d: R \rightarrow R$ with the property $d(x y x)=$ $d(x) y^{*} x^{*}+x d(y) x^{*}+x y d(x)$ for all $x, y \in R$. It could easily be seen that any Jordan $*$-derivation on a 2 -torsion free $*$-ring is a Jordan triple $*$-derivation [6, Lemma 2]. Vukman [7] has proved that any Jordan triple $*$-derivation on a 6 -torsion free semiprime $*$-ring is a Jordan $*$-derivation.

Let $\mathbb{N}_{0}$ be the set of all nonnegative integers and let $D=$ $\left(d_{i}\right)_{i \in \mathbb{N}_{0}}$ be a family of additive mappings of a ring $R$ such that $d_{0}=i d_{R}$. Then $D$ is said to be a higher derivation (resp., a Jordan higher derivation) of $R$ if, for each $n \in \mathbb{N}_{0}, d_{n}(x y)=$ $\sum_{i+j=n} d_{i}(x) d_{j}(y)$ (resp., $\left.d_{n}\left(x^{2}\right)=\sum_{i+j=n} d_{i}(x) d_{j}(x)\right)$ holds for all $x, y \in R$. The concept of higher derivations was introduced by Hasse and Schmidt [8]. This interesting notion of higher derivations has been studied in both commutative and noncommutative rings; see, for example, [9-13]. Clearly, every higher derivation is a Jordan higher derivation. Ferrero and Haetinger [13] have extended Herstein's theorem [2] for higher derivations on 2-torsion free semiprime rings. For an account of higher and Jordan higher derivations the reader is referred to [14]. A family $D=\left(d_{i}\right)_{i \in \mathbb{N}_{0}}$ of additive mappings of a ring $R$, where $d_{0}=i d_{R}$, is called a Jordan triple higher derivation if $d_{n}(x y x)=\sum_{i+j+k=n} d_{i}(x) d_{j}\left(y^{i}\right) d_{k}\left(x^{i+j}\right)$ holds for 
all $x, y \in R$. Ferrero and Haetinger [13] have proved that every Jordan higher derivation of a 2-torsion free ring is a Jordan triple higher derivation. They also have proved that every Jordan triple higher derivation of a 2-torsion free semiprime ring is a higher derivation.

Motivated by the notions of $*$-derivations and higher derivations, we naturally introduce the notions of higher *derivations, Jordan higher *-derivations, and Jordan triple higher $*$-derivations. Our main objective in this paper is to show that every Jordan triple higher *-derivation of a 6torsion free semiprime $*$-ring is a Jordan higher $*$-derivation. This result extends the main result of [7]. It is also shown that every Jordan higher $*$-derivation of a 2 -torsion free $*$-ring is a Jordan triple higher $*$-derivation. So we can conclude that the notions of Jordan triple higher $*$-derivations and Jordan higher $*$-derivations are coincident on 6-torsion free semiprime $*$-rings.

\section{Preliminaries and Main Results}

We begin by the following definition.

Definition 1. Let $\mathbb{N}_{0}$ be the set of all nonnegative integers and let $D=\left(d_{i}\right)_{i \in \mathbb{N}_{0}}$ be a family of additive mappings of a $*$-ring $R$ such that $d_{0}=i d_{R}$. $D$ is called

(a) a higher $*$-derivation of $R$ if, for each $n \in \mathbb{N}_{0}$,

$$
d_{n}(x y)=\sum_{i+j=n} d_{i}(x) d_{j}\left(y^{*^{i}}\right) \quad \forall x, y \in R ;
$$

(b) a Jordan higher $*$-derivation of $R$ if, for each $n \in \mathbb{N}_{0}$,

$$
d_{n}\left(x^{2}\right)=\sum_{i+j=n} d_{i}(x) d_{j}\left(x^{*^{i}}\right) \quad \forall x \in R
$$

(c) a Jordan triple higher $*$-derivation of $R$ if, for each $n \in$ $\mathbb{N}_{0}$,

$$
d_{n}(x y x)=\sum_{i+j+k=n} d_{i}(x) d_{j}\left(y^{*^{i}}\right) d_{k}\left(x^{*^{i+j}}\right) \quad \forall x, y \in R
$$
tion.

Throughout this section, we will use the following nota-

Notation. Let $D=\left(d_{i}\right)_{i \in \mathbb{N}_{0}}$ be a Jordan triple higher $*$ derivation of a $*$-ring $R$. For every fixed $n \in \mathbb{N}_{0}$ and each $x, y \in R$, we denote by $A_{n}(x)$ and $B_{n}(x, y)$ the elements of $R$ defined by

$$
\begin{aligned}
A_{n}(x)= & d_{n}\left(x^{2}\right)-\sum_{i+j=n} d_{i}(x) d_{j}\left(x^{*^{i}}\right), \\
B_{n}(x, y)= & d_{n}(x y+y x)-\sum_{i+j=n} d_{i}(x) d_{j}\left(y^{*^{i}}\right) \\
& -\sum_{i+j=n} d_{i}(y) d_{j}\left(x^{*^{i}}\right) .
\end{aligned}
$$

It can easily be seen that $A_{n}(-x)=A_{n}(x), B_{n}(-x, y)=$ $-B_{n}(x, y)$, and $A_{n}(x+y)=A_{n}(x)+A_{n}(y)+B_{n}(x, y)$ for each pair $x, y \in R$. We will use these relations without any explicit mention in the steps of the proofs. The next lemmas are crucial in developing the proofs of the main results.

Lemma 2 (see [5, Lemma 1.1]). Let $R$ be a 2-torsion free semiprime ring. If $x, y \in R$ are such that $x r y+y r x=0$ for all $r \in R$, then $x r y=y r x=0$ for all $r \in R$. If $R$ is semiprime, then $x r y=0$ for all $r \in R$ implies $y r x=x y=y x=0$.

Lemma 3 (see [7, Lemma 1]). Let $R$ be a 2-torsion free semiprime $*$-ring. If $x, y \in R$ are such that $x r^{*} y^{*}+y r x=0$ for all $r \in R$, then $x y=y x=0$.

Lemma 4. Let $D=\left(d_{i}\right)_{i \in \mathbb{N}_{0}}$ be a Jordan triple higher $*$ derivation of $a *$-ring $R$. If $A_{m}(x)=0$ for all $x \in R$ and for each $m \leq n$, then $A_{n}(x) y^{*^{n}} x^{2^{*^{n}}}+x^{2} y A_{n}(x)=0$ for each $n \in \mathbb{N}_{0}$ and for every $x, y \in R$.

Proof. The substitution of $x y x$ for $y$ in the definition of Jordan triple higher $*$-derivation gives

$$
\begin{aligned}
& d_{n}(x(x y x) x) \\
& =\sum_{i+j+k=n} d_{i}(x) d_{j}\left((x y x)^{*^{i}}\right) d_{k}\left(x^{*^{i+j}}\right) \\
& =\sum_{i+j+k=n} d_{i}(x)\left(\sum_{p+q+r=j} d_{p}\left(x^{*^{i}}\right) d_{q}\left(y^{*^{i+p}}\right) d_{r}\left(x^{*^{i+p+q}}\right)\right) \\
& \times d_{k}\left(x^{*^{i+j}}\right){ }^{i+p+q+r+k=n} d_{i}(x) d_{p}\left(x^{*^{i}}\right) d_{q}\left(y^{*^{i+p}}\right) d_{r}\left(x^{*^{i+p+q}}\right) \\
& \times d_{k}\left(x^{*^{i+p+q+r}}\right) \\
& =\sum_{i+p=n} d_{i}(x) d_{p}\left(x^{*^{i}}\right) y^{*^{n}} x^{*^{n}} x^{*^{n}}+x^{2} y \sum_{r+k=n} d_{r}(x) d_{k}\left(x^{*^{r}}\right) \\
& +\sum_{\substack{i+p+q+r+k=n \\
i+p \neq n, r+k \neq n}} d_{i}(x) d_{p}\left(x^{*^{i}}\right) d_{q}\left(y^{*^{i+p}}\right) d_{r}\left(x^{*^{i+p+q}}\right) \\
& \left.+x_{k}^{*^{i+p+q+r}}\right) .
\end{aligned}
$$

On the other hand, the substitution of $x^{2}$ for $x$ in the definition of Jordan triple higher $*$-derivation and using our assumption that $A_{m}(x)=0$ for $m<n$ give

$$
\begin{aligned}
& d_{n}\left(x^{2} y x^{2}\right) \\
& \quad=\sum_{i+j+k=n} d_{i}\left(x^{2}\right) d_{j}\left((y)^{*^{i}}\right) d_{k}\left(x^{2^{*^{i+j}}}\right)
\end{aligned}
$$




$$
\begin{aligned}
& =d_{n}\left(x^{2}\right) y^{*^{n}} x^{*^{n}} x^{*^{n}}+x^{2} y d_{n}\left(x^{2}\right) \\
& +\sum_{\substack{i+j+k=n \\
i \neq n, k \neq n}} d_{i}\left(x^{2}\right) d_{j}\left((y)^{*^{i}}\right) d_{k}\left(x^{2^{*^{i+j}}}\right) \\
& =d_{n}\left(x^{2}\right) y^{*^{n}} x^{*^{n}} x^{*^{n}}+x^{2} y d_{n}\left(x^{2}\right) \\
& +\sum_{\substack{i+j+k=n \\
i \neq n, k \neq n}}\left(\sum_{u+v=i} d_{u}(x) d_{v}\left(x^{*^{u}}\right)\right) d_{j}\left(y^{*^{i}}\right) \\
& \times\left(\sum_{s+t=k} d_{s}\left(x^{*^{i+j}}\right) d_{t}\left(x^{*^{i+j+s}}\right)\right) \\
& =d_{n}\left(x^{2}\right) y^{*^{n}} x^{*^{n}} x^{*^{n}}+x^{2} y d_{n}\left(x^{2}\right) \\
& +\sum_{\substack{u+v+j+s+t=n \\
u+v \neq n, s+t \neq n}} d_{u}(x) d_{v}\left(x^{*^{u}}\right) d_{j}\left(y^{*^{u+v}}\right) d_{s} \\
& \times\left(x^{*^{u+v+j}}\right) d_{t}\left(x^{*^{u+v+j+s}}\right) .
\end{aligned}
$$

Now, subtracting the two relations so obtained we find that

$$
\begin{aligned}
& \left(d_{n}\left(x^{2}\right)-\sum_{i+p=n} d_{i}(x) d_{p}\left(x^{*^{i}}\right)\right) y^{*^{n}} x^{2^{*^{n}}} \\
& +x^{2} y\left(d_{n}\left(x^{2}\right)-\sum_{r+k=n} d_{r}(x) d_{k}\left(x^{*^{r}}\right)\right)=0 .
\end{aligned}
$$

Using our notation the last relation reduces to the required result.

Now, we are ready to prove our main results.

Theorem 5. Let $R$ be a 6-torsion free semiprime *-ring. Then every Jordan triple higher $*$-derivation $D=\left(d_{i}\right)_{i \in \mathbb{N}_{0}}$ of $R$ is a Jordan higher $*$-derivation of $R$.

Proof. We intend to show that $A_{n}(x)=0$ for all $x \in R$. In case $n=0$, we get trivially $A_{0}(x)=0$ for all $x \in R$. If $n=1$, then it follows from [7, Theorem 1] that $A_{1}(x)=0$ for all $x \in R$. Thus we assume that $A_{m}(x)=0$ for all $x \in R$ and $m<n$. Thus, from Lemma 4, we see that

$$
A_{n}(x) y^{*^{n}} x^{2^{*^{n}}}+x^{2} y A_{n}(x)=0 \quad \forall x, y \in R
$$

In case $n$ is even, (8) reduces to $A_{n}(x) y x^{2}+x^{2} y A_{n}(x)=0$; by applying Lemma 2 we get $A_{n}(x) x^{2}=x^{2} A_{n}(x)=0$. In case $n$ is odd, (8) reduces to $A_{n}(x) y^{*} x^{2^{*}}+x^{2} y A_{n}(x)=0$; by applying Lemma 3 we get $A_{n}(x) x^{2}=x^{2} A_{n}(x)=0$. So for either of the two cases we have for each $n$

$$
\begin{array}{ll}
A_{n}(x) x^{2}=0 & \forall x \in R, \\
x^{2} A_{n}(x)=0 & \forall x \in R .
\end{array}
$$

The substitution of $x+y$ for $x$ in relation (9) gives

$$
\begin{gathered}
A_{n}(x) y^{2}+A_{n}(y) x^{2}+B_{n}(x, y) x^{2}+B_{n}(x, y) y^{2} \\
+A_{n}(x)(x y+y x)+A_{n}(y)(x y+y x) \\
+B_{n}(x, y)(x y+y x)=0 \quad \forall x, y \in R .
\end{gathered}
$$

Substituting $-x$ for $x$ in (11) we obtain

$$
\begin{aligned}
A_{n}(x) & y^{2}+A_{n}(y) x^{2}-B_{n}(x, y) x^{2} \\
& -B_{n}(x, y) y^{2}-A_{n}(x)(x y+y x) \\
& -A_{n}(y)(x y+y x) \\
& +B_{n}(x, y)(x y+y x)=0 \quad \forall x, y \in R .
\end{aligned}
$$

Comparing (11) and (12) we get, since $R$ is 2-torsion free, that

$$
\begin{gathered}
B_{n}(x, y) x^{2}+B_{n}(x, y) y^{2}+A_{n}(x)(x y+y x) \\
+A_{n}(y)(x y+y x)=0 \quad \forall x, y \in R .
\end{gathered}
$$

Putting $2 x$ for $x$ in (13) gives by the assumption that $R$ is 2 torsion free that

$$
\begin{gathered}
4 B_{n}(x, y) x^{2}+B_{n}(x, y) y^{2}+4 A_{n}(x)(x y+y x) \\
+A_{n}(y)(x y+y x)=0 \quad \forall x, y \in R .
\end{gathered}
$$

Subtracting the relation (13) from (14) we obtain, since $R$ is 3-torsion free, that

$$
B_{n}(x, y) x^{2}+A_{n}(x)(x y+y x)=0 \quad \forall x, y \in R .
$$

Right multiplication of (15) by $A_{n}(x) x$ and using (9) we obtain

$$
A_{n}(x) x y A_{n}(x) x+A_{n}(x) y x A_{n}(x) x=0 \quad \forall x, y \in R .
$$

Putting $y x$ for $y$ in (16) and left-multiplying by $x$ we get $\left(x A_{n}(x) x\right) y\left(x A_{n}(x) x\right)=0$, for all $x, y \in R$. By the semiprimeness of $R$ it follows that $x A_{n}(x) x=0$ for all $x \in R$. So (16) reduces to $A_{n}(x) x y A_{n}(x) x=0$, for all $x, y \in R$. Again, by the semiprimeness of $R$, we get

$$
A_{n}(x) x=0 \quad \forall x \in R .
$$

Using (17), (15) reduces to $B_{n}(x, y) x^{2}+A_{n}(x) y x=0$ for all $x, y \in R$. Multiplying this relation by $A(x)$ from the right and by $x$ from the left we get $x A_{n}(x) y x A_{n}(x)=0$ for all $x, y \in R$. Again, by the semiprimeness of $R$, we get

$$
x A_{n}(x)=0 \quad \forall x \in R .
$$

Linearizing (17) we have

$$
\begin{aligned}
& A_{n}(x) y+B_{n}(x, y) x+A_{n}(y) x \\
& +B_{n}(x, y) y=0 \quad \forall x, y \in R .
\end{aligned}
$$


Putting $-x$ for $x$ in (19) we get

$$
\begin{array}{r}
A_{n}(x) y+B_{n}(x, y) x-A_{n}(y) x \\
-B_{n}(x, y) y=0 \quad \forall x, y \in R .
\end{array}
$$

Adding (19) and (20) we get, since $R$ is 2-torsion free, that

$$
A_{n}(x) y+B_{n}(x, y) x=0 \quad \forall x, y \in R
$$

Multiplying (21) by $A_{n}(x)$ from the right and using (18) we get $A_{n}(x) y A_{n}(x)=0$ for all $x, y \in R$. By the semiprimeness of $R$, we get $A_{n}(x)=0$ for all $x \in R$. This completes the proof of the theorem.

Corollary 6 (see [7, Theorem 1]). Let $R$ be a 6-torsion free semiprime $*$-ring. Then every Jordan triple $*$-derivation of $R$ is a Jordan $*$-derivation of $R$.

Theorem 7. Let $R$ be a 2-torsion free *-ring. Then every Jordan higher $*$-derivation $D=\left(d_{i}\right)_{i \in \mathbb{N}_{0}}$ of $R$ is a Jordan triple higher $*$-derivation of $R$.

Proof. We have

$$
d_{n}\left(x^{2}\right)=\sum_{i+j=n} d_{i}(x) d_{j}\left(x^{*^{i}}\right) .
$$

Put $v=x+y$ and using (22) we obtain

$$
\begin{aligned}
d_{n}\left(v^{2}\right)= & \sum_{i+j=n} d_{i}(x+y) d_{j}\left((x+y)^{*^{i}}\right) \\
= & \sum_{i+j=n}\left(d_{i}(x) d_{j}\left(x^{*^{i}}\right)+d_{i}(y) d_{j}\left(y^{*^{i}}\right)\right. \\
& \left.+d_{i}(x) d_{j}\left(y^{*^{i}}\right)+d_{i}(y) d_{j}\left(x^{*^{i}}\right)\right), \\
d_{n}\left(v^{2}\right)= & d_{n}\left(x^{2}+x y+y x+y^{2}\right) \\
= & d_{n}\left(x^{2}\right)+d_{n}\left(y^{2}\right)+d_{n}(x y+y x) \\
= & \sum_{l+m=n} d_{l}(x) d_{m}\left((x)^{*^{l}}\right) \\
& +\sum_{r+s=n} d_{r}(y) d_{s}\left((y)^{*^{r}}\right)+d_{n}(x y+y x) .
\end{aligned}
$$

Comparing the last two forms of $d_{n}\left(v^{2}\right)$ gives

$$
d_{n}(x y+y x)=\sum_{i+j=n}\left(d_{i}(x) d_{j}\left(y^{*^{i}}\right)+d_{i}(y) d_{j}\left(x^{*^{i}}\right)\right) .
$$

Now put $w=x(x y+y x)+(x y+y x) x$. Using (24) we get

$$
\begin{aligned}
d_{n}(w)= & \sum_{i+j=n} d_{i}(x) d_{j}\left((x y+y x)^{*^{i}}\right) \\
& +\sum_{i+j=n} d_{i}(x y+y x) d_{j}\left(x^{*^{i}}\right) \\
= & \sum_{i+j=n} \sum_{r+s=j} d_{i}(x) d_{r}\left(x^{*^{i}}\right) d_{s}\left(y^{*^{i+r}}\right) \\
& +\sum_{i+j=n} \sum_{r+s=j} d_{i}(x) d_{r}\left(y^{*^{i}}\right) d_{s}\left(x^{*^{i+r}}\right) \\
& +\sum_{i+j=n} \sum_{k+l=i} d_{k}(x) d_{l}\left(y^{*^{k}}\right) d_{j}\left(x^{*^{k+l}}\right) \\
& +\sum_{i+j=n} \sum_{k+l=i} d_{k}(y) d_{l}\left(x^{*^{k}}\right) d_{j}\left(x^{*^{k+l}}\right) \\
= & \sum_{i+r+s=n} d_{i}(x) d_{r}\left(x^{*^{i}}\right) d_{s}\left(y^{*^{*+r}}\right) \\
& +2 \sum_{i+j+k=n} d_{i}(x) d_{j}\left(y^{*^{i}}\right) d_{k}\left(x^{*^{i+j}}\right) \\
& +\sum_{k+l+j=n}(y) d_{l}\left(x^{*^{k}}\right) d_{j}\left(x^{*^{k+l}}\right) . \\
& \left.+2{ }^{2}\right)
\end{aligned}
$$

Also,

$$
\begin{aligned}
d_{n}(w)= & d_{n}\left(\left(x^{2} y+y x^{2}\right)+2 x y x\right) \\
= & d_{n}\left(x^{2} y+y x^{2}\right)+2 d_{n}(x y x) \\
= & 2 d_{n}(x y x)+\sum_{r+s+j=n} d_{r}(x) d_{s}\left(x^{*^{r}}\right) d_{j}\left(y^{*^{r+s}}\right) \\
& +\sum_{i+k+l=n} d_{i}(y) d_{k}\left(x^{*^{i}}\right) d_{l}\left(x^{*^{i+k}}\right) .
\end{aligned}
$$

Comparing the last two forms of $d_{n}(w)$ and using the fact that $R$ is 2 -torsion free, we obtain the required result.

By Theorems 5 and 7, we can state the following.

Theorem 8. The notions of Jordan higher *-derivation and Jordan triple higher $*$-derivation on a 6-torsion free semiprime *-ring are coincident.

\section{Conflict of Interests}

The author declares that there is no conflict of interests regarding the publication of this paper.

\section{Acknowledgments}

The author is truly indebted to Professor M. N. Daif for his constant encouragement and valuable discussions. The author also would like to express sincere gratitude to the 
referees for their careful reading and helpful comments. This paper is a part of the author's Ph.D. dissertation under the supervision of Professor M. N. Daif.

\section{References}

[1] M. Ashraf, S. Ali, and C. Haetinger, "On derivations in rings and their applications," The Aligarh Bulletin of Mathematics, vol. 25, no. 2, pp. 79-107, 2006.

[2] I. N. Herstein, "Jordan derivations of prime rings," Proceedings of the American Mathematical Society, vol. 8, pp. 1104-1110, 1957.

[3] M. Brešar, "Jordan derivations on semiprime rings," Proceedings of the American Mathematical Society, vol. 104, no. 4, pp. 10031006, 1988.

[4] I. N. Herstein, Topics in Ring Theory, The University of Chicago Press, Chicago, Ill, USA, 1969.

[5] M. Brešar, "Jordan mappings of semiprime rings," Journal of Algebra, vol. 127, no. 1, pp. 218-228, 1989.

[6] M. Brešar and J. Vukman, "On some additive mappings in rings with involution," Aequationes Mathematicae, vol. 38, no. 2-3, pp. 178-185, 1989.

[7] J. Vukman, "A note on Jordan *-derivations in semiprime rings with involution," International Mathematical Forum, vol. 1, no. 13-16, pp. 617-622, 2006.

[8] H. Hasse and F. K. Schmidt, "Noch eine begrüdung der theorie der höheren differential quotienten in einem algebraaischen funktionenkorper einer unbestimmeten," Journal für die Reine und Angewandte Mathematik, vol. 177, pp. 215-237, 1937.

[9] L. N. Macarro, "On the modules of $m$-integrable derivations in non-zero characteristic," Advances in Mathematics, vol. 229, no. 5, pp. 2712-2740, 2012.

[10] D. Hoffmann and P. Kowalski, "Integrating Hasse-Schmidt derivations," 2012, http://arxiv.org/abs/1212.5788.

[11] Z. Xiao and F. Wei, "Jordan higher derivations on triangular algebras," Linear Algebra and its Applications, vol. 432, no. 10, pp. 2615-2622, 2010.

[12] F. Wei and Z. Xiao, "Generalized Jordan derivations on semiprime rings and its applications in range inclusion problems," Mediterranean Journal of Mathematics, vol. 8, no. 3, pp. 271-291, 2011.

[13] M. Ferrero and C. Haetinger, "Higher derivations and a theorem by Herstein," Quaestiones Mathematicae. Journal of the South African Mathematical Society, vol. 25, no. 2, pp. 249-257, 2002.

[14] C. Haetinger, M. Ashraf, and S. Ali, "On higher derivations: a survey," International Journal of Mathematics, Game Theory, and Algebra, vol. 19, no. 5-6, pp. 359-379, 2011. 


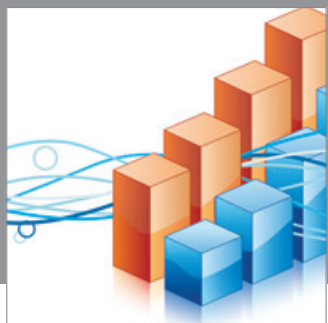

Advances in

Operations Research

mansans

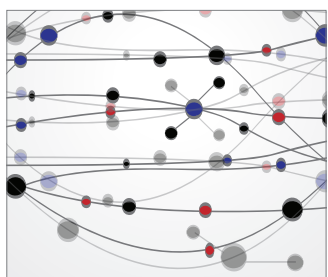

The Scientific World Journal
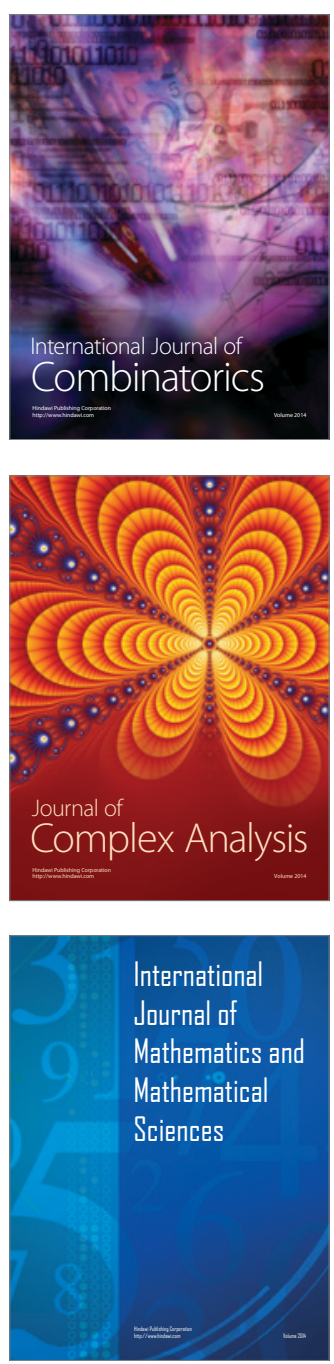
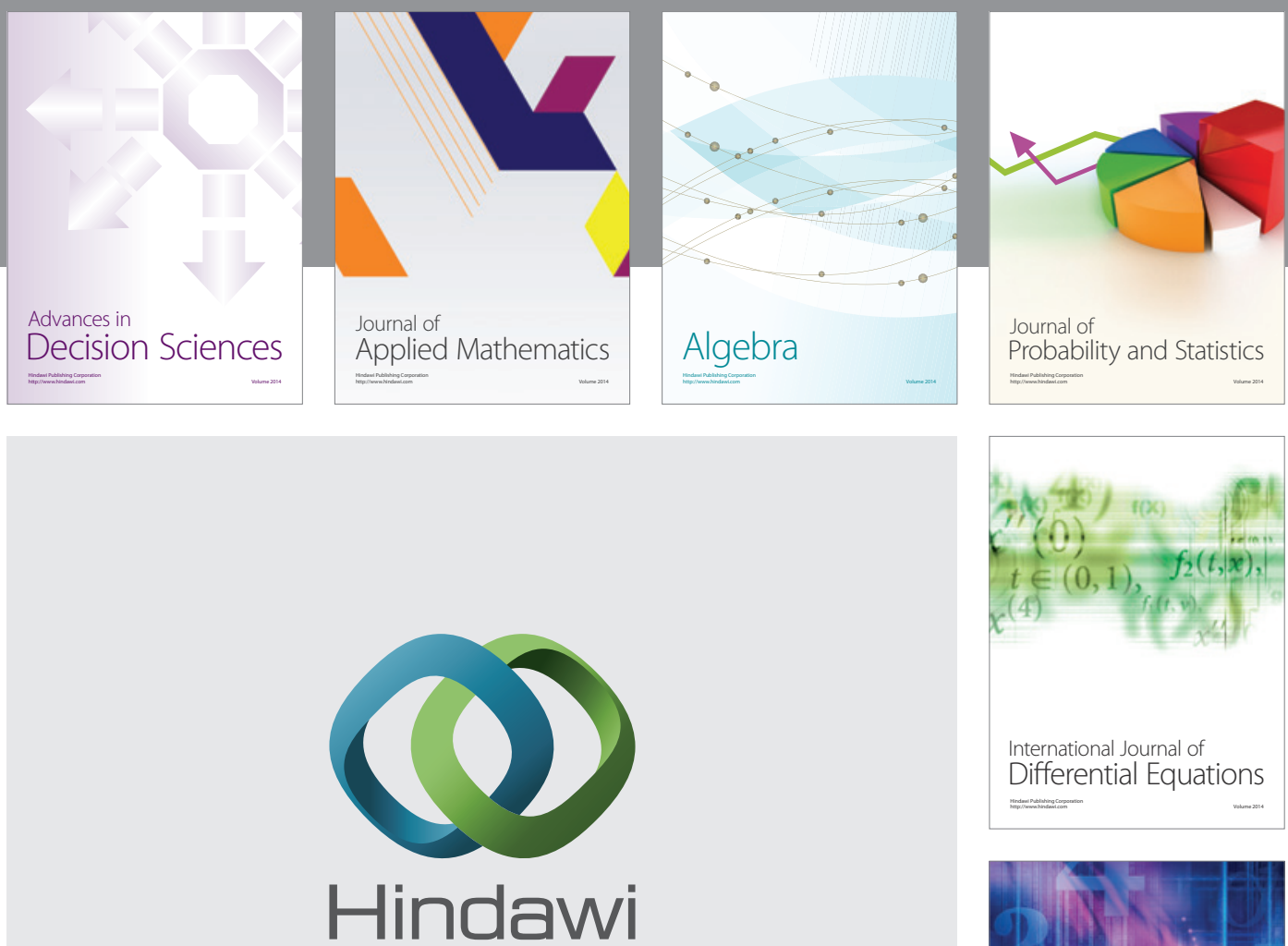

Submit your manuscripts at http://www.hindawi.com
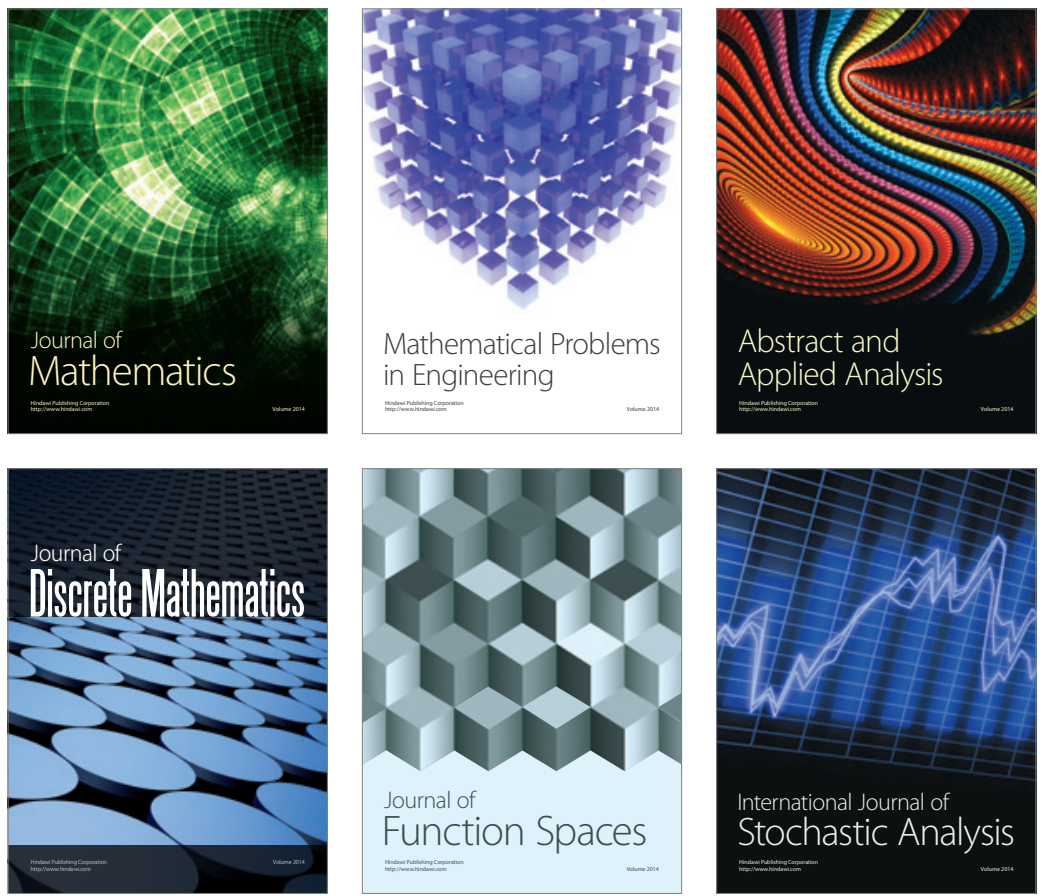

Journal of

Function Spaces

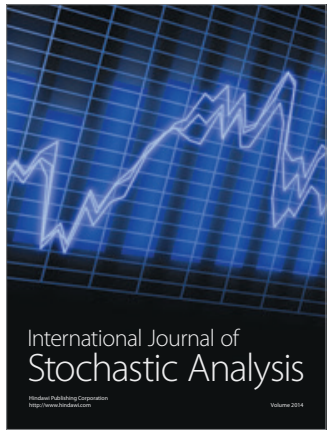

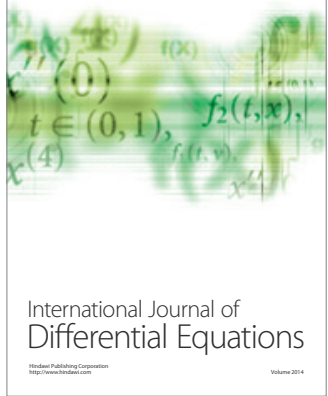
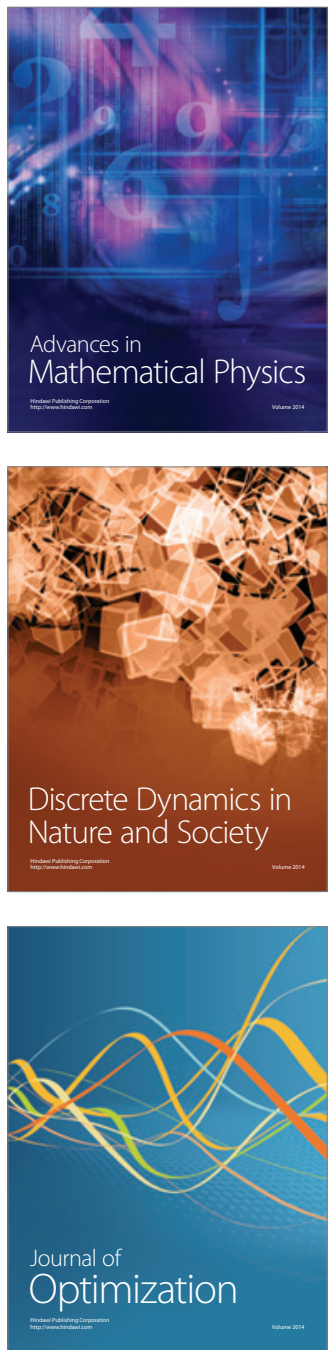